\title{
A Constructive Analysis of Convex-Valued Demand Correspondence for Weakly Uniformly Rotund and Monotonic Preference
}

\author{
Yasuhito Tanaka ${ }^{1}$ and Atsuhiro Satoh ${ }^{2}$ \\ ${ }^{1}$ Faculty of Economics, Doshisha University, Kamigyo-ku, Kyoto 602-8580, Japan \\ ${ }^{2}$ Graduate School of Economics, Doshisha University, Kamigyo-ku, Kyoto 602-8580, Japan \\ Correspondence should be addressed to Yasuhito Tanaka, yasuhito@mail.doshisha.ac.jp
}

Received 23 December 2010; Accepted 14 February 2011

Academic Editor: Chenghu Ma

Copyright (C) 2011 Y. Tanaka and A. Satoh. This is an open access article distributed under the Creative Commons Attribution License, which permits unrestricted use, distribution, and reproduction in any medium, provided the original work is properly cited.

Bridges (1992) has constructively shown the existence of continuous demand function for consumers with continuous, uniformly rotund preference relations. We extend this result to the case of multivalued demand correspondence. We consider a weakly uniformly rotund and monotonic preference relation and will show the existence of convex-valued demand correspondence with closed graph for consumers with continuous, weakly uniformly rotund and monotonic preference relations. We follow the Bishop style constructive mathematics according to Bishop and Bridges (1985), Bridges and Richman (1987), and Bridges and Vîţă (2006).

\section{Introduction}

Bridges ([1]) has constructively shown the existence of continuous demand function for consumers with continuous, uniformly rotund preference relations. We extend this result to the case of multivalued demand correspondence. We consider a weakly uniformly rotund and monotonic preference relation and will show the existence of convex-valued demand correspondence with closed graph for consumers with continuous, weakly uniformly rotund and monotonic preference relations.

In the next section, we summarize some preliminary results most of which were proved in [1]. In Section 3, we will show the main result.

We follow the Bishop style constructive mathematics according to [2-4].

\section{Preliminary Results}

Consider a consumer who consumes $N$ goods. $N$ is a finite natural number larger than 1 . Let $X \subset R^{N}$ be his consumption set. It is a compact (totally bounded and complete) and convex 
set. Let $\Delta$ be an $n$-1-dimensional simplex and $p \in \Delta$ a normalized price vector of the goods. Let $p_{i}$ be the price of the $i$ th good, then $\sum_{i=1}^{N} p_{i}=1$ and $p_{i} \geq 0$ for each $i$. For a given $p$, the budget set of the consumer is

$$
\beta(p, w) \equiv\{x \in X: p \cdot x \leq w\}
$$

where $w>0$ is the initial endowment. A preference relation of the consumer $>$ is a binary relation on $X$. Let $x, y \in X$. If he prefers $x$ to $y$, we denote $x>y$. A preference-indifference relation $\succsim$ is defined as follows;

$$
x \succsim y, \quad \text { iff } \neg(y>x),
$$

where $x>y$ entails $x \succsim y$, the relations $>$ and $\succsim$ are transitive, and if either $x \succsim y>z$ or $x>y \succsim z$, then $x>z$. Also we have

$$
x \succsim y \quad \text { iff } \forall z \in X(y>z \Longrightarrow x>z) .
$$

A preference relation $>$ is continuous if it is open as a subset of $X \times X$, and $\succsim$ is a closed subset of $X \times X$.

A preference relation $>$ on $X$ is uniformly rotund if for each $\varepsilon$ there exists a $\delta(\varepsilon)$ with the following property.

Definition 2.1 (uniformly rotund preference). Let $\varepsilon>0, x$, and $y$ points of $X$ such that $|x-y| \geq$ $\varepsilon$, and $z$ a point of $R^{N}$ such that $|z| \leq \delta(\varepsilon)$, then either $(1 / 2)(x+y)+z>x$ or $(1 / 2)(x+y)+z>y$.

Strict convexity of preference is defined as follows.

Definition 2.2 (strict convexity of preference). If $x, y \in X, x \neq y$, and $0<t<1$, then either $t x+(1-t) y>x$ or $t x+(1-t) y>y$. convex.

Bridges [5] has shown that if a preference relation is uniformly rotund, then it is strictly

On the other hand, convexity of preference is defined as follows.

Definition 2.3 (convexity of preference). If $x, y \in X, x \neq y$, and $0<t<1$, then either $t x+(1-$ $t) y \succsim x$ or $t x+(1-t) y \succsim y$.

We define the following weaker version of uniform rotundity.

Definition 2.4 (weakly uniformly rotund preference). Let $\varepsilon>0, x$ and $y$ points of $X$ such that $|x-y| \geq \varepsilon$. Let $z$ be a point of $R^{N}$ such that $|z| \leq \delta$ for $\delta>0$ and $z \gg 0$ (every component of $z$ is positive), then $(1 / 2)(x+y)+z>x$ or $(1 / 2)(x+y)+z>y$.

We assume also that consumers' preferences are monotonic in the sense that if $x^{\prime}>x$ (it means that each component of $x^{\prime}$ is larger than or equal to the corresponding component of $x$, and at least one component of $x^{\prime}$ is larger than the corresponding component of $x$ ), then $x^{\prime}>x$. 
Now, we show the following lemmas.

Lemma 2.5. If $x, y \in X, x \neq y$, then weak uniform rotundity of preferences implies that $(1 / 2)(x+$ $y) \succsim x$ or $(1 / 2)(x+y) \succsim y$.

Proof. Consider a decreasing sequence $\left(\delta_{m}\right)$ of $\delta$ in Definition 2.4. Then, either $(1 / 2)(x+y)+$ $z_{m}>x$ or $(1 / 2)(x+y)+z_{m}>y$ for $z_{m}$ such that $\left|z_{m}\right|<\delta_{m}$ and $z_{m} \gg 0$ for each $m$. Assume that $\left(\delta_{m}\right)$ converges to zero. Then, $(1 / 2)(x+y)+z_{m}$ converges to $(1 / 2)(x+y)$. Continuity of the preference (closedness of $\succsim)$ implies that $(1 / 2)(x+y) \succsim x$ or $(1 / 2)(x+y) \succsim y$.

Lemma 2.6. If a consumer's preference is weakly uniformly rotund, then it is convex.

This is a modified version of Proposition 2.2 in [5].

Proof. (1) Let $x$ and $y$ be points in $X$ such that $|x-y| \geq \varepsilon$. Consider a point $(1 / 2)(x+y)$. Then, $|x-(1 / 2)(x+y)| \geq \varepsilon / 2$ and $|(1 / 2)(x+y)-y| \geq \varepsilon / 2$. Thus, using Lemma 2.5, we can show $(1 / 4)(3 x+y) \succsim x$ or $(1 / 4)(3 x+y) \succsim y$, and $(1 / 4)(x+3 y) \succsim x$ or $(1 / 4)(x+3 y) \succsim$ $y$. Inductively, we can show that for $k=1,2, \ldots, 2^{n}-1,\left(k / 2^{n}\right) x+\left(\left(2^{n}-k\right) / 2^{n}\right) y \succsim x$ or $\left(k / 2^{n}\right) x+\left(\left(2^{n}-k\right) / 2^{n}\right) y \succsim y$, for each natural number $n$.

(2) Let $z=t x+(1-t) y$ with a real number $t$ such that $0<t<1$. We can select a natural number $k$ so that $k / 2^{n} \leq t \leq(k+1) / 2^{n}$ for each natural number $n$. $\left((k+1) / 2^{n}-k / 2^{n}\right)=\left(1 / 2^{n}\right)$ is a sequence. Since, for natural numbers $m$ and $n$ such that $m>n, l / 2^{m} \leq t \leq(l+1) / 2^{m}$ and $k / 2^{n} \leq t \leq(k+1) / 2^{n}$ with some natural number $l$, we have

$$
\left|\left(\frac{l+1}{2^{m}}-\frac{l}{2^{m}}\right)-\left(\frac{k+1}{2^{n}}-\frac{k}{2^{n}}\right)\right|=\left|\frac{2^{n}-2^{m}}{2^{m} 2^{n}}\right|<\frac{1}{2^{n}}
$$

$\left((k+1) / 2^{n}-k / 2^{n}\right)$ is a Cauchy sequence, and converges to zero. Then, $\left((k+1) / 2^{n}\right)$ and $\left(k / 2^{n}\right)$ converge to $t$. Closedness of $\succsim$ implies that either $z \succsim x$ or $z \succsim y$. Therefore, the preference is convex.

Lemma 2.7. Let $x$ and $y$ be points in $X$ such that $x>y$. Then, if a consumer's preference is weakly uniformly rotund and monotonic, $t x+(1-t) y>y$ for $0<t<1$.

Proof. By continuity of the preference (openness of $>$ ), there exists a point $x^{\prime}=x-\lambda$ such that $\lambda \gg 0$ and $x^{\prime}>y$. Then, since weak uniform rotundity implies convexity, we have $t x^{\prime}+(1-t) y \succsim y$ or $t x^{\prime}+(1-t) y \succsim x^{\prime}$. If $t x^{\prime}+(1-t) y \succsim x^{\prime}$, then by transitivity $t x^{\prime}+(1-t) y=$ $t x+(1-t) y-t \lambda \succsim x^{\prime}>y$. Monotonicity of the preference implies $t x+(1-t) y>y$. Assume $t x^{\prime}+(1-t) y \succsim y$. Then, again monotonicity of the preference implies $t x+(1-t) y>y$.

Let $S$ be a subset of $\Delta \times R$ such that for each $(p, w) \in S$,

(1) $p \in \Delta$,

(2) $\beta(p, w)$ is nonempty,

(3) There exists $\xi \in X$ such that $\xi>x$ for all $x \in \beta(p, w)$.

In [1], the following lemmas were proved.

Lemma 2.8 ([1, Lemma 2.1]). If $p \in \Delta \subset R^{N}, w \in R$, and $\beta(p, w)$ is nonempty, then $\beta(p, w)$ is compact. 
Lemma 2.8 with Proposition 4.4 in Chapter 4 of [2] or Proposition 2.2.9 of [4] implies that for each $(p, w) \in S \beta(p, w)$ is located in the sense that the distance

$$
\rho(x, \beta(p, w)) \equiv \inf \{|x-y|: y \in \beta(p, w)\}
$$

exists for each $x \in R^{N}$.

Lemma 2.9 ([1, Lemma 2.2]). If $(p, w) \in S$ and $\xi>\beta(p, w)$ (it means $\xi>x$, for all $x \in \beta(p, w)$ ), then $\rho(\xi, \beta(p, w))>0$ and $p \cdot \xi>w$.

Lemma 2.10 ([1, Lemma 2.3]). Let $(p, c) \in S, \xi \in X$ and $\xi>\beta(p, c)$. Let $H$ be the hyperplane with equation $p \cdot x=c$. Then, for each $x \in \beta(p, c)$, there exists a unique point $\varphi(x)$ in $H \cap[x, \xi]$. The function $\varphi$ so defined maps $\beta(p, c)$ onto $H \cap \beta(p, c)$ and is uniformly continuous on $\beta(p, c)$.

Lemma 2.11 ([1, Lemma 2.4]). Let $(p, w) \in S, r>0, \xi \in X$, and $\xi>\beta(p, w)$. Then, there exists $\zeta \in X$ such that $\rho(\zeta, \beta(p, w))<r$ and $\zeta>\beta(p, w)$.

Proof. See the appendix.

And the following lemma.

Lemma 2.12 ([1, Lemma 2.8]). Let $R, c$, and $t$ be positive numbers. Then, there exists $r>0$ with the following property: if $p, p^{\prime}$ are elements of $R^{N}$ such that $|p| \geq c$ and $\left|p-p^{\prime}\right|<r, w$, w $w^{\prime}$ are real numbers such that $\left|w-w^{\prime}\right|<r$, and $y^{\prime}$ is an element of $R^{N}$ such that $\left|y^{\prime}\right| \leq R$ and $p^{\prime} \cdot y^{\prime}=w^{\prime}$, then there exists $\zeta \in R^{N}$ such that $p \cdot \zeta=w$ and $\left|y^{\prime}-\zeta\right|<t$.

It was proved by setting $r=c t /(R+1)$.

\section{Convex-Valued Demand Correspondence with Closed Graph}

With the preliminary results in the previous section, we show the following our main result.

Theorem 3.1. Let $\succsim$ be a weakly uniformly rotund preference relation on a compact and convex subset $X$ of $R^{N}, \Delta$ a compact and convex set of normalized price vectors (an $n$-1-dimensional simplex), and $S$ a subset of $\Delta \times R$ such that for each $(p, w) \in S$

(1) $p \in \Delta$,

(2) $\beta(p, w)$ is nonempty,

(3) There exists $\xi \in X$ such that $\xi>x$ for all $x \in \beta(p, w)$.

Then, for each $(p, w) \in S$, there exists a subset $F(p, w)$ of $\beta(p, w)$ such that $F(p, w) \succsim x$ (it means $y \succsim x$ for all $y \in F(p, w))$ for all $x \in \beta(p, w), p \cdot F(p, w)=w(p \cdot y=w$ for all $y \in F(p, w))$, and the multivalued correspondence $F(p, w)$ is convex-valued and has a closed graph.

A graph of a correspondence $F(p, w)$ is

$$
G(F)=\bigcup_{(p, w) \in S}(p, w) \times F(p, w)
$$

If $G(F)$ is a closed set, we say that $F$ has a closed graph. 
Proof. (1) Let $(p, w) \in S$, and choose $\xi \in X$ such that $\xi>\beta(p, w)$. By Lemma 2.11, construct a sequence $\left(\zeta_{m}\right)$ in $X$ such that $\zeta_{m}>\beta(p, w)$ and $\rho\left(\zeta_{m}, \beta(p, w)\right)<\left(r / 2^{m-1}\right)$ with $r>0$ for each natural number $m$. By convexity and transitivity of the preference $t \zeta_{m}+(1-t) \zeta_{m+1}>\beta(p, w)$ for $0<t<1$ and each $m$. Thus, we can construct a sequence $\left(\zeta_{n}\right)$ such that $\left|\zeta_{n}-\zeta_{n+1}\right|<$ $\varepsilon^{n}, \rho\left(\zeta_{n}, \beta(p, w)\right)<\delta^{n}$ and $\zeta_{n}>\beta(p, w)$ for some $0<\varepsilon<1$ and $0<\delta<1$, and so $\left(\zeta_{n}\right)$ is a Cauchy sequence in $X$. It converges to a limit $\zeta^{*} \in X$. By continuity of the preference (closedness of $\succsim) \zeta^{*} \succsim \beta(p, w)$, and $\rho\left(\zeta^{*}, \beta(p, w)\right)=0$. Since $\beta(p, w)$ is closed, $\zeta^{*} \in \beta(p, w)$. By Lemma 2.9, $p \cdot \zeta_{n}>w$ for all $n$. Thus, we have $p \cdot \zeta^{*}=w$. Convexity of the preference implies that $\zeta^{*}$ may not be unique, that is, there may be multiple elements $\zeta^{\prime}$ of $\beta(p, w)$ such that $p \cdot \zeta^{\prime}=w$ and $\zeta^{\prime} \succsim \beta(p, w)$. Therefore, $F(p, w)$ is a set and we get a demand correspondence. Let $\zeta \in F(p, w)$ and $\zeta^{\prime} \in F(p, w)$. Then, $\zeta \succsim \beta(p, w), \zeta^{\prime} \succsim \beta(p, w)$, and convexity of the preference implies $t \zeta+(1-t) \zeta^{\prime} \succsim \beta(p, w)$. Thus, $F(p, w)$ is convex.

(2) Next, we prove that the demand correspondence has a closed graph. Consider $(p, w)$ and $\left(p^{\prime}, w^{\prime}\right)$ such that $\left|p-p^{\prime}\right|<r$ and $\left|w-w^{\prime}\right|<r$ with $r>0$. Let $F(p, w)$ and $F\left(p^{\prime}, w^{\prime}\right)$ be demand sets. Let $y^{\prime} \in F\left(p^{\prime}, w^{\prime}\right), c=\rho(0, \Delta)>0$, and $R>0$ such that $X \subset \bar{B}(0, R)$. Given $\varepsilon>0, t=\delta>0$ such that $\delta<\varepsilon$, and choose $r$ as in Lemma 2.12. By that lemma, we can choose $\zeta \in R^{N}$ such that $p \cdot \zeta=w$ and $\left|y^{\prime}-\zeta\right|<\delta$. Similarly, we can choose $\zeta^{\prime}(y) \in R^{N}$ such that $p^{\prime} \cdot \zeta^{\prime}(y)=w^{\prime}$ and $\left|y-\zeta^{\prime}(y)\right|<\delta$ for each $y \in F(p, w) \cdot y^{\prime} \in F\left(p^{\prime}, w^{\prime}\right)$ means $y^{\prime} \succsim \zeta^{\prime}(y)$. Either $\left|y^{\prime}-y\right|>\varepsilon / 2$ for all $y \in F(p, w)$ or $\left|y^{\prime}-y\right|<\varepsilon$ for some $y \in F(p, w)$. Assume that $\left|y^{\prime}-y\right|>\varepsilon / 2$ for all $y \in F(p, w)$ and $y>\zeta$. If $\delta$ is sufficiently small, $\left|y^{\prime}-y\right|>\varepsilon / 2$ means $|y-\zeta|>\varepsilon / k$ and $\left|y^{\prime}-\zeta^{\prime}(y)\right|>\varepsilon / k$ for some finite natural number $k$. Then, by weak uniform rotundity, there exist $z_{n}$ and $z_{n}^{\prime}$ such that $\left|z_{n}\right|<\tau_{n},\left|z_{n}^{\prime}\right|<\tau_{n}$ with $\tau_{n}>0, z_{n} \gg 0$ and $z_{n}^{\prime} \gg 0,(1 / 2)(y+\zeta)+z_{n}>\zeta$ and $(1 / 2)\left(y^{\prime}+\zeta^{\prime}(y)\right)+z_{n}^{\prime}>\zeta^{\prime}(y)$ for $n=1,2, \ldots$ Again if $\delta$ is sufficiently small, $\left|y-\zeta^{\prime}(y)\right|<\delta$ and $\left|y^{\prime}-\zeta\right|<\delta$ imply $(1 / 2)(y+\zeta)+z_{n}>y^{\prime}$ and $(1 / 2)\left(y^{\prime}+\zeta^{\prime}(y)\right)+z_{n}^{\prime}>y$. And it follows that $\left|(1 / 2)(y+\zeta)-(1 / 2)\left(y^{\prime}+\zeta^{\prime}(y)\right)\right|<\delta$. By continuity of the preference (openness of $>$ ) $(1 / 2)(y+\zeta)+z_{n}^{\prime}>y$. Let $y_{1}=(1 / 2)(y+\zeta)$. Consider a sequence $\left(\tau_{n}\right)$ converging to zero. By continuity of the preference (closedness of $\succsim) ~ y_{1} \succsim y^{\prime}$ and $y_{1} \succsim y$. Note that $p \cdot y_{1}=w$. Thus, $y_{1} \in \beta(p, w)$. Since $y \in F(p, w)$, we have $y_{1} \in F(p, w)$. Replacing $y$ with $y_{1}$, we can show that $(y+3 \zeta) / 4 \in F(p, w)$. Inductively, we obtain $\left(y+\left(2^{m}-1\right) \zeta\right) / 2^{m} \in F(p, w)$ for each natural number $m$. Then, we have $|y-\zeta|<\eta$ for some $y \in F(p, w)$ for any $\eta>0$. It contradicts $|y-\zeta|>\varepsilon / k$. Therfore, we have $\left|y^{\prime}-y\right|<\varepsilon$ or $\zeta \succsim y$ (it means $|y-\zeta|<\delta+\varepsilon$ and $\zeta \in F(p, w)$ ), and so $F(p, w)$ has a closed graph.

\section{Appendix}

\section{A. Proof of Lemma 2.11}

This proof is almost identical to the proof of Lemma 2.4 in Bridges [1]. They are different in a few points.

Let $H$ be the hyperplane with equation $p \cdot x=w$ and $\xi^{\prime}$ the projection of $\xi$ on $H$. Assume $\left|\xi-\xi^{\prime}\right|>3 r$. Choose $R$ such that $H \cap \beta(p, w)$ is contained in the closed ball $\bar{B}\left(\xi^{\prime}, R\right)$ around $\xi^{\prime}$ and let

$$
c=\sqrt{1+\left(\frac{R}{\left|\xi-\xi^{\prime}\right|}\right)^{2}} .
$$




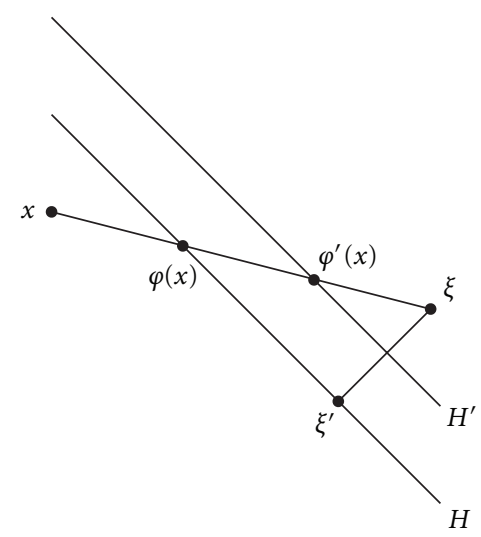

Figure 1: Calculation of $\left|\varphi(x)-\varphi^{\prime}(x)\right|$.

Let $H^{\prime}$ be the hyperplane parallel to $H$, between $H$ and $\xi$ and a distance $r / 2 c$ from $H$, and $H^{\prime \prime}$ the hyperplane parallel to $H$, between $H$ and $\xi$ and a distance $r / c$ from $H$. For each $x \in \beta(p, w)$ let $\varphi(x)$ be the unique element of $H \cap[x, \xi], \varphi^{\prime}(x)$ the unique element of $H^{\prime} \cap[x, \xi]$, and $\varphi^{\prime \prime}(x)$ the unique element of $H^{\prime \prime} \cap[x, \xi]$. Since $\xi>\beta(p, w)$, we have $\varphi^{\prime \prime}(x)>\varphi(x) \succsim x$ by convexity and continuity of the preference. $\varphi^{\prime}(x)$ is uniformly continuous, so

$$
T \equiv\left\{\varphi^{\prime}(x): x \in \beta(p, w)\right\}
$$

is totally bounded by Lemma 2.8 and Proposition 4.2 in Chapter 4 of [2].

Since $\varphi^{\prime \prime}(x)>\varphi(x)$ and $\varphi^{\prime}(x)=(1 / 2) \varphi^{\prime \prime}(x)+(1 / 2) \varphi(x)$, we have $\varphi^{\prime}(x)>x$, and so continuity of the preference (openness of $>$ ) means that there exists $\delta>0$ such that $\varphi^{\prime}\left(x_{i}\right)>x$ when $\left|\varphi^{\prime}\left(x_{i}\right)-\varphi^{\prime}(x)\right|<\delta$. Let $\left(x_{1}, \ldots, x_{n}\right)$ be points of $\beta(p, w)$ such that $\left(\varphi^{\prime}\left(x_{1}\right), \ldots, \varphi^{\prime}\left(x_{n}\right)\right)$ is a $\delta$-approximation to $T$. Given $x$ in $\beta(p, w)$, choose $i$ such that $\left|\varphi^{\prime}\left(x_{i}\right)-\varphi^{\prime}(x)\right|<\delta$. Then, $\varphi^{\prime}\left(x_{i}\right)>x$.

Now, from our choice of $c$, we have $\left|\varphi(x)-\varphi^{\prime}(x)\right|<r / 2$ for each $x \in \beta(p, w)$. It is proved as follows. Since by the assumption $\left|\varphi(x)-\xi^{\prime}\right|<R,|\varphi(x)-\xi|<\sqrt{R^{2}+\left|\xi-\xi^{\prime}\right|^{2}}$. Thus, we have

$$
\left|\varphi(x)-\varphi^{\prime}(x)\right|<\frac{r}{2 c} \times \frac{\sqrt{R^{2}+\left|\xi-\xi^{\prime}\right|^{2}}}{\left|\xi-\xi^{\prime}\right|}=\frac{r}{2 c} \sqrt{1+\left(\frac{R}{\left|\xi-\xi^{\prime}\right|}\right)^{2}}=\frac{r}{2} .
$$

See Figure 1.

Let

$$
\begin{gathered}
t_{1}=1-\frac{r}{2 n\left|\varphi^{\prime}\left(x_{1}\right)-\xi\right|} \\
\eta_{1}=t_{1} \varphi^{\prime}\left(x_{1}\right)+\left(1-t_{1}\right) \xi .
\end{gathered}
$$

Then, $\left|\eta_{1}-\varphi^{\prime}\left(x_{1}\right)\right|=r / 2 n, \rho\left(\eta_{1}, \beta(p, w)\right)<r(n+1) / 2 n$ (because $\left|\varphi\left(x_{1}\right)-\varphi^{\prime}\left(x_{1}\right)\right|<r / 2$ and $\left.\varphi\left(x_{1}\right) \in \beta(p, w)\right)$, and by convexity of the preference $\eta_{1} \succsim \xi$ or $\eta_{1} \succsim \varphi^{\prime}\left(x_{1}\right)$. 
In the first case, we complete the proof by taking $\zeta=\eta_{1}$. In the second, assume that, for some $k(1 \leq k \leq n-1)$, we have constructed $\eta_{1}, \ldots, \eta_{k}$ in $X$ such that

$$
\begin{gathered}
\eta_{k} \succsim \varphi^{\prime}\left(x_{i}\right) \quad(1 \leq i \leq k), \\
\rho\left(\eta_{k}, \beta(p, w)\right)<\frac{r(n+k)}{2 n} .
\end{gathered}
$$

As $\left|\xi-\eta_{k}\right|>r$ (because $\left|\xi-\xi^{\prime}\right|>3 r$ ), we can choose $y \in\left[\eta_{k}, \xi\right]$ such that $\left|y-\eta_{k}\right|=r / 2 n$. Then, $\rho(y, \beta(p, w))<r(n+k+1) / 2 n$ and either $y \succsim \xi$ or $y \succsim \eta_{k}$. In the former case, the proof is completed by taking $\zeta=y$. If $y \succsim \eta_{k}, y+\lambda / 2>\eta_{k}-\lambda / 2$ for all $\lambda$ such that $\lambda \gg 0$. Then, either $y+\lambda / 2>\varphi^{\prime}\left(x_{k+1}\right)$ for all $\lambda$ and so $y \succsim \varphi^{\prime}\left(x_{k+1}\right)$, in which case we set $\eta_{k+1}=y$; or else $\varphi^{\prime}\left(x_{k+1}\right)>\eta_{k}-\lambda / 2$ for all $\lambda$ and so $\varphi^{\prime}\left(x_{k+1}\right) \succsim \eta_{k}$, then we set $\eta_{k+1}=\varphi^{\prime}\left(x_{k+1}\right)$.

If this process proceeds as far as the construction of $\eta_{n}$, then, setting $\zeta=\eta_{n}$, we see that $\rho(\zeta, \beta(p, w))<r$ and that $\zeta \succsim \varphi^{\prime}\left(x_{i}\right)$ for each $i$; so $\zeta>x$ for each $x \in \beta(p \cdot w)$.

\section{Acknowledgment}

This research was partially supported by the Ministry of Education, Science, Sports and Culture of Japan, Grant-in-Aid for Scientific Research (C), no. 20530165, and the Special Costs for Graduate Schools of the Special Expenses for Hitech Promotion by the Ministry of Education, Science, Sports and Culture of Japan in 2010.

\section{References}

[1] D. S. Bridges, "The construction of a continuous demand function for uniformly rotund preferences," Journal of Mathematical Economics, vol. 21, no. 3, pp. 217-227, 1992.

[2] E. Bishop and D. Bridges, Constructive Analysis, vol. 279 of Grundlehren der Mathematischen Wissenschaften, Springer, Berlin, Germany, 1985.

[3] D. Bridges and F. Richman, Varieties of Constructive Mathematics, vol. 97 of London Mathematical Society Lecture Note Series, Cambridge University Press, Cambridge, UK, 1987.

[4] D. Bridges and L. Vîţă, Techniques of Constructive Mathematics, Springer, 2006.

[5] D. S. Bridges, "Constructive notions of strict convexity," Mathematical Logic Quarterly, vol. 39, no. 3, pp. 295-300, 1993. 


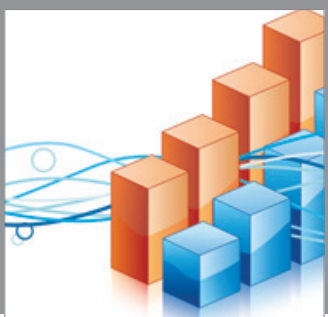

Advances in

Operations Research

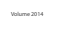

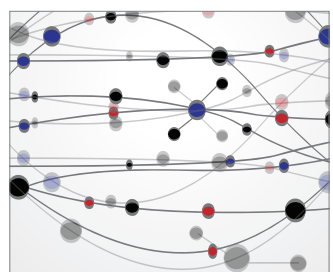

\section{The Scientific} World Journal
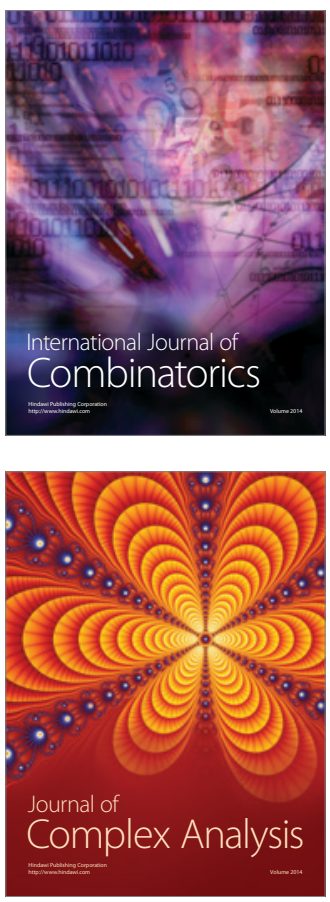

International Journal of

Mathematics and

Mathematical

Sciences
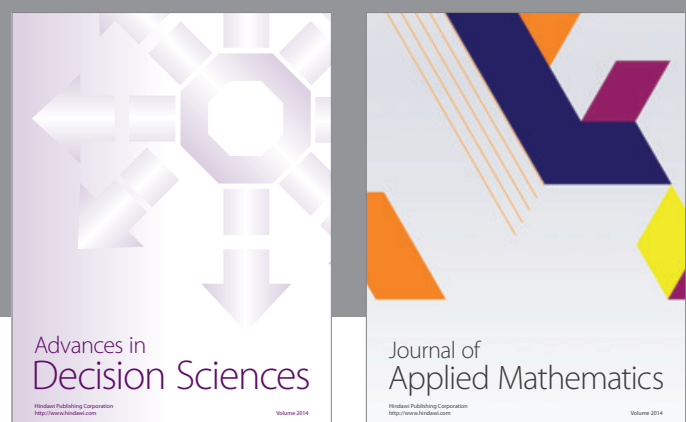

Journal of

Applied Mathematics
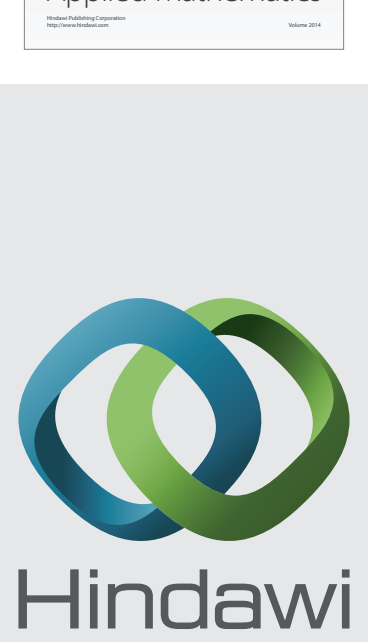

Submit your manuscripts at http://www.hindawi.com
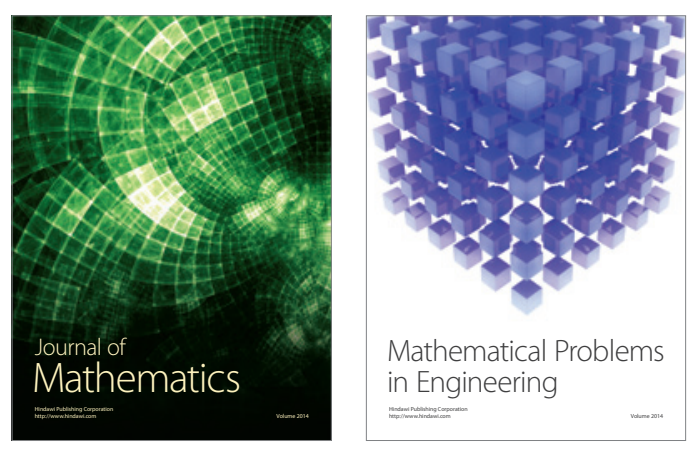

Mathematical Problems in Engineering
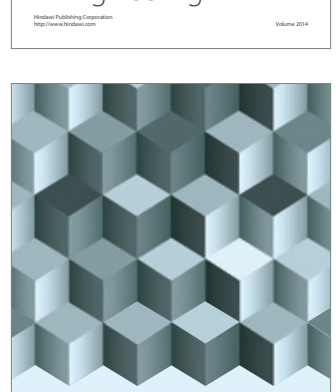

Journal of

Function Spaces
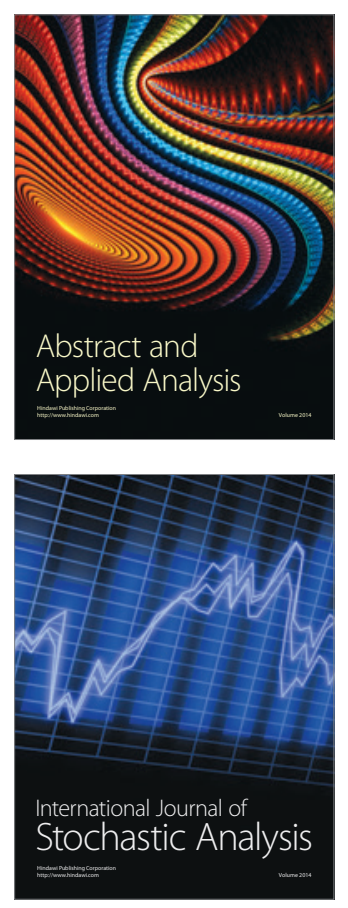

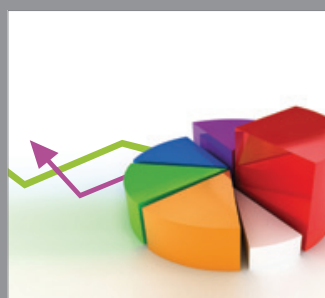

ournal of

Probability and Statistics

Promensencen
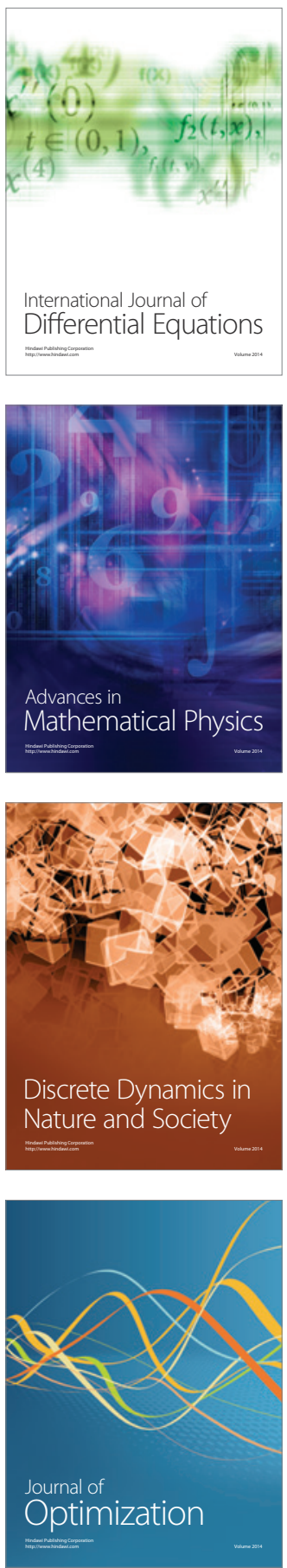